\title{
AN INFINITE PACKING THEOREM FOR SPHERES
}

\author{
OSCAR WESLER
}

By removing a sequence of disjoint circles from a given circle in such a way that only a set of measure zero remains behind, Wolff in 1921 was able to give an example of a function defined by a series of rational fractions which could be extended analytically into the domain of the "apparent poles," thereby settling a doubt that had been raised by Denjoy, Borel and other investigators in the theory of functions of a complex variable [4]. Reflecting upon this example, Borel [1] was led to observe that as a consequence of one of his own theorems on analytic extensions the areas of such disjoint circles must necessarily form a convergent series whose convergence is less rapid than that of the series with general term $e^{-e k^{2}}$, and to wonder further what the order of magnitude of these circles might be, and whether one could determine it directly. It turned out that Borel's bound was incredibly weak: the convergence is actually so much slower that the radii of these circles can be shown to form a divergent series! That is to say, to state it simply for the unit circle, if the radii $\left\{r_{k}\right\}$ of the disjoint circles satisfy the condition $\sum_{k=1}^{\infty} r_{k}^{2}=1$, then $\sum_{k=1}^{\infty} r_{k}=\infty$. A proof of this, using the machinery of uniform approximations to functions of a complex variable, can be found in Mergelyan [3]. In this paper we give a direct and simple proof of this geometric result. In fact, our method is such that it yields at once a result of greater generality: we shall show that the infinite packing theorem just mentioned holds not only for circles in the plane, but that an analogous result holds for spheres in $n$-space, as well as for more general figures.

TheOREM. Let $\left\{C_{k}\right\}$ with radii $\left\{r_{k}\right\}, k=1,2,3, \cdots$, be a sequence of disjoint spheres in $n$-space contained in the unit sphere $C_{0}$ and exhausting it but for a set of measure zero, i.e. so that $\sum_{\boldsymbol{k}=1}^{\infty} r_{\boldsymbol{k}}^{n}=1$. Then $\sum_{k=1}^{\infty} r_{k}^{n-1}=\infty$.

Proof. For $n=1$, the theorem is trivially true. For $n \geqq 2$, let $C_{0}^{\prime}$ be the $(n-1)$-dimensional sphere obtained as the intersection of $C_{0}$ with any hyperplane through its center, and let $\left\{C_{k}^{\prime}\right\}$ be the sequence of possibly overlapping spheres in $C_{0}^{\prime}$ got by projecting the $C_{k}$ orthogonally onto $C_{0}^{\prime}$. Consider the chords of $C_{0}$ perpendicular to $C_{0}^{\prime}$. Our method will consist in showing that almost every such chord necessarily meets infinitely many of the disjoint spheres $C_{k}$ in $C_{0}$, so that almost every point of $C_{0}^{\prime}$ is contained in infinitely many of

Received by the editors June 29, 1959. 
the spheres $C_{k}^{\prime}$. It would then follow at once from the easier half of the well-known Borel-Cantelli lemma (see, e.g. [2]) that the sum of the $(n-1)$-dimensional volumes of the spheres $C_{k}^{\prime}$ is infinite, equivalently, that $\sum_{\mathbf{k}=1}^{\infty} r_{\mathbf{k}}^{n-1}=\infty$.

For each point $x \in C_{0}^{\prime}$, therefore, let $L(x)$ be the chord of $C_{0}$ perpendicular to $C_{0}^{\prime}$ at $x$. Let $E$ denote the set of $n$-dimensional Lebesgue measure zero, referred to in the statement of the theorem, that remains behind in $C_{0}$ after the $C_{k}$ are removed. Let $\mu_{m}$ denote ordinary $m$-dimensional Lebesgue measure and let $M=\left\{x \mid \mu_{1}(L(x) \cap E)>0\right\}$. It follows at once from $\mu_{n}(E)=0$ that $\mu_{n-1}(M)=0$. Consider now only those points $x \in C_{0}^{\prime}-M$. Suppose for such an $x$ that the chord $L(x)$ meets only finitely many of the spheres $C_{k}$. Since $\mu_{1}(L(x) \cap E)$ $=0, L(x)$ must clearly be completely covered by these finitely many spheres, hence $L(x)$ must pass successively from one sphere to another in this finite string of spheres through points of tangency. Since there are at most countably many possible points of tangency among $C_{0}$ and the $C_{k}$, and therefore only countably many finite subsets of these points of tangency, it follows that there are at most only countably many such chords $L(x)$ meeting only finitely many spheres, so that for the corresponding set $N$ of such points $x$ we have $\mu_{n-1}(N)=0$. But this means that for all points $x \in C_{0}^{\prime}-(M \cup N)$, i.e. for almost every point $x$ of $C_{0}^{\prime}$, the chord $L(x)$ meets infinitely many of the disjoint spheres $C_{k}$, which completes the proof.

We conclude this paper with two observations upon the hypothesis of the theorem and the nature of the result.

First, the question invariably arises whether it is indeed possible to so fill up or pack a sphere to within a subset of measure zero by means of a sequence of disjoint subspheres, and this question is readily answered in the affirmative. For example, to see it for circles in the plane, let $C_{1}$ be any circle interiorly tangent to $C_{0}$ and let $C_{2}$ be the largest circle in $C_{0}-C_{1}$. At this first stage it is easily checked that with $C_{1}$ and $C_{2}$ we have removed at least $1 / 2$ the area of $C_{0}$, leaving two "triangular" regions behind in $C_{0}$. By further removing the largest tangent circle and then the next largest if necessary and so on a finite number of times from each of these two triangular regions, it is easily seen that we can remove at least $1 / 2$ the remaining area, leaving behind at this second stage at most $1 / 4$ the original area, consisting of a finite collection of triangular regions, each determined by three mutually tangent circles. Continuing in this way for each of these triangular regions (removing one circle per region will usually do) we see that at the $k$ th stage of this procedure the area of the remaining set goes to zero with $(1 / 2)^{k}$. For spheres in $n$-space, another 
method may be used, as follows. Cover $C_{0}$ interiorly to within any small fraction of its volume by means of a fine cubical grid and then remove the largest sphere from within each of these cubicles. The volume of an $n$-sphere with radius $r$ being equal to $2 \pi^{n / 2} r^{n} / n \Gamma(n / 2)$, and that of a cube with side $2 r$ being $(2 r)^{n}$, we have thus removed approximately the proper fraction $\epsilon_{n}=\pi^{n / 2} / n 2^{n-1} \Gamma(n / 2)$ of the volume. By using a much finer grid within the remaining set, again removing spheres from the cubicles, and continuing in this way stage after stage, we see that the volume of the set left behind is made to go to zero with $\left(1-\epsilon_{n}\right)^{k}$ as $k$ goes to infinity.

Second, as to the statement and conclusion of the theorem, it should be observed, by way of further generalization, that neither the nature of the result nor the method of proof depends in any essential way upon the fact of spheres or upon the uniqueness of their points of tangency. When more general figures are involved, one abandons the convenient formulation of the result in terms of radii and restates it in terms of areas and perimeters, or volumes and surface areas. Nor is it essential that sets of measure zero remain behind. Given any method of packing a region $C_{0}$ with a sequence of disjoint subregions $C_{k}$, which is such that the orthogonally projected images $C_{k}^{\prime}$ of the $C_{k}$ upon some fixed hyperplane satisfy the condition $\mu_{n-1}\left(\lim \sup C_{k}^{\prime}\right)>0$, it follows by the Borel-Cantelli lemma that $\sum_{k=1}^{\infty} \mu_{n-1}\left(C_{k}^{\prime}\right)=\infty$, a fortiori, that $\sum_{k=1}^{\infty} \mu_{n-1}$ (surface $C_{k}$ ) $=\infty$. The result holds, for example, for a polygon packed to within a set of measure zero by disjoint ellipses, for squares in a circle, for spheres in a cube, for an ellipsoid packed to within a set of measure zero by disjoint polyhedra (a simple modification of the above argument on the chords $L(x)$ in the neighborhood of the boundary of the ellipsoid is required to show the countability of the exceptional chords), and so on. We omit the details.

\section{BIBLIOGRAPHY}

1. Emile Borel, Remarques sur la note de M. J. Wolff, C. R. Acad. Sci. Paris vol. 173 (1921) pp. 1057-1059.

2. Michel Loève, Probability theory, New York, D. Van Nostrand, 1955, p. 228.

3. S. N. Mergelyan, Uniform approximations to functions of a complex variable, Uspehi Mat. Nauk. vol. 7 (1952) pp. 31-122; Amer. Math. Soc. Transl. no. 101 (1954) p. 21.

4. J. Wolff, Sur les séries $\sum A_{k} /\left(z-\alpha_{k}\right)$, C. R. Acad. Sci. Paris vol. 173 (1921) pp. 1056-1057.

University of Michigan 